\title{
SOME PROPERTIES OF FUNCTIONALS ON LEVEL SETS
}

\author{
R.G. SALAKHUDINOV
}

\begin{abstract}
In the paper we consider special functionals on a planar domain $G$ constructed by means of the distance to the boundary $\partial G$ and a classical warping function. The functionals depending on the distance function are considered for simply-connected domains. We also study the functionals depending on the warping function for a finite-connected domain. We prove that the property of isoperimetric monotonicity with respect to a free parameter gives rise to another monotonicity, namely, the monotonicity of the functionals considered as the functions of the sets defined on subsets of the domain. Some partial cases of the inequality were earlier obtained by Payne. We note that the inequalities were successfully applied for justifying new estimates for the torsional rigidity of simply-connected and multiply-connected domains. In particular, we construct new functionals of domains monotone in both its variables. Moreover, we find sharp estimates of variation rate of the functions, that is, we obtain sharp estimates of their derivatives.
\end{abstract}

Keywords: distance to boundary, warping function, Payne type inequality, isoperimetric inequality, isoperimetric monotonicity.

Mathematics Subject Classification: Primary 28A25, 35A23; Secondary 30A10

\section{INTRODUCTION}

To solve effectively some problems of mathematical physics, it is not sufficient to know such classical geometric characteristics of a domain like the area, volume, area of surface, length of the boundary, diameter, the maximum of radii of the circles contained in the domain. A more gentle and effective tool for solving some problems are Euclidean momenta of the domain with respect to its boundary.

Let $G$ be a simply-connected domain in the plane. An Euclidean momentum of order $\alpha$ with respect to the boundary of the domain $G$ is the functional

$$
\mathbf{I}_{\alpha}(G):=\int_{G} \rho(x, G)^{\alpha} \mathrm{dA}
$$

where $\rho(x, G)$ is the distance from a point $x \in G$ to the boundary $\partial G, \alpha>-1$ and dA is the area differential. It follows from work [1] that under an appropriate normalization, functional (1.1) ranges between the length of the boundary and the maximal radius of a circle contained in the domain. Each of these values can be attained under an appropriate choice of the parameter.

Euclidean momenta arise in mathematical physics while estimating various physical functionals of a domain. For instance, as it was shown by F.G. Avkhadiev [2], the Euclidean inertia momentum of a domain $(\alpha=2)$ and the torsional rigidity of a domain are comparable quantities on the class of simply-connected domains, that is, the quotient of the functionals is bounded from above and below by positive finit constants independent of the domain. Euclidean momenta of various order arise in studying Hardy type inequalities in a multi-dimensional Euclidean space, see, for instance, [3]. We note that multi-dimensional analogues of functional (1.1) are employed in solving variational problems, see [4, 5].

R.G. Salakhudinov, Some Properties of Functionals on LEVEl Sets.

(C)SAlakHudinovR.G. 2019.

The work is supported by RFBR (project no. 17-01-00282-a) and by the subsidy appropriated to Kazan

Federal University for performing state task in the scientific activity (1.9773.2017/8.9).

Submitted September 26, 2017. 
Application of isoperimetric inequalities on the level sets often leads one to solving various problems and to new methods. A bright example of such kind is the application of a classical isoperimetric inequality in theory of torsion and theory of flow of a viscous liquid, see [6]; we also mention its important role in the symmetrization methods, see [7, 8].

One of the aims of our work is to study the properties of functional (1.1) on the subsets of $G$. We note that in work [9], stationary Euclidean momenta $(\alpha=1)$ and Euclidean inertia momenta of subdomains were employed to estimate the torsional rigidity of a domain. On the other hand, a passage to the limit from the level sets allows one to consider the estimates on these sets as a generalization of inequalities between functionals of the domain. For instance, our main statements imply that a series of classical inequalities (Saint-Venant-Pólya inequality, Payne inequality and others) can be obtained by passing to the limit from the level set of a classical warping function and the distance to the boundary of a domain.

It was shown in works [1, 9, 10 that the Euclidean momenta of order $\alpha$ and $L^{p}$-norms of the warping function possess a series of similar isoperimetric properties. Following this similarity, in this work we consider similar issue of the case, when the function $\rho(x, G)$ in $(1.1)$ is replaced by the classical warping function $u(x, G)$ and in this case the domain $G$ is finitely-connected on the plane.

The main idea of obtaining inequalities for the functionals is the application of Payne type inequalities [6, 11] on the level sets of functions.

\section{DISCUSSION OF PROBLEMS AND PRELIMINARY RESULTS}

We introduce notations we shall need. Let

$$
\begin{aligned}
G(\mu) & :=\{x \in G \mid \rho(x, G)>\mu\}, \quad \mathbf{a}(\mu) \equiv \mathbf{a}(G(\mu)):=\int_{G(\mu)} \mathrm{dA} . \\
\mathbf{l}(\mu) & \equiv \mathbf{L}(G(\mu)):=\int_{\partial G(\mu)} \mathrm{d} s .
\end{aligned}
$$

In what follows, the sets $G(\mu)$ are called level sets of the function $\rho(x, G)$.

We consider the following geometric functional

$$
\mathbf{f}_{\alpha}(\mu):=\mathbf{I}_{\alpha}(G(\mu)),
$$

where $0 \leqslant \mu \leqslant \boldsymbol{\rho}(G), \boldsymbol{\rho}(G):=\sup _{x \in G} \rho(x, G)$ and $\alpha$ is a real parameter. For a fixed $\mu$, the structure of the dependence of the function $\mathbf{f}_{\alpha}(\mu)$ on the parameter $\alpha$ has a isoperimetric nature. We are interesting in the properties of the function $\mathbf{f}_{\alpha}(\mu)$ for a fixed value of the parameter. We shall show that the value of the parameter and the geometry of the domain $G$ determine many properties of the function $\mathbf{f}_{\alpha}(\mu)$.

Let $\mathbf{I}_{\alpha_{0}}(G)<+\infty$ for some $\alpha_{0}>-1$ and for the smaller values of the parameter the functional be unbounded. Then all sets of level $G(\mu)$ have a bounded are except possibly the set of zero level, that is, of the area of the domain $G$, see, for instance, [12]. It is known that if the area of the domain is bounded and $\alpha \geqslant 0$, then the inequality holds:

$$
\mathbf{I}_{\alpha}(G) \leqslant \frac{\mathbf{A}(G)^{1+\frac{\alpha}{2}}}{\pi^{\frac{\alpha}{2}}(\alpha+1)(\alpha+2)} .
$$

If we apply the latter inequality to $G(\mu)$, then the function $\mathbf{f}_{\alpha}(\mu)$ is well-defined and finite as $\mu \in(0, \boldsymbol{\rho}(G)]$ and $\alpha \geqslant 0$.

As $0>\alpha_{0}>-1$, the area of the domain is finite and hence, the same holds for the area of level sets. But as we shall show below, calculation of $\mathbf{f}_{\alpha}(\mu)$ for negative values of the parameter is closely related with the properties of the functional $\mathbf{l}(\mu)$ as a function of $\mu$.

We note that the restriction $\alpha>-1$ is natural while considering the functional $\mathbf{I}_{\alpha}(G)$ and the condition $\mathbf{I}_{\alpha_{0}}(G)<+\infty$ describes a certain class of domains on the plane; moreover, different values of the parameter correspond to different classes. 
It is natural to consider the value of the function $\mathbf{f}_{\alpha}(\mu)$ at $\mu=0$ as a result of the passage to the limit. Then as $\alpha \geqslant \alpha_{0}$, the point $\mu=0$ can be included in the domain, while for $\alpha \leqslant \alpha_{0}$ we just let $\mathbf{f}_{\alpha}(0):=+\infty$. As it was mentioned above, the value of the parameter $\alpha=\alpha_{0}$ does not play such role for $\mathbf{f}_{\alpha}(\mu)$.

On the other hand, the condition $\boldsymbol{\rho}(G)<+\infty$ is necessary for studying functional (1.1) and also sufficient for studying e function (2.2). The case $\alpha=+\infty$ correspond to the class of domains with $\boldsymbol{\rho}(G)<+\infty$. The examples of a strip and a half-strip disappoint us since the function $\mathbf{f}_{\alpha}(\mu)$ is identically infinite on its domain. But it is easy to construct examples, when only from some value $\mu_{0}$ the areas of the level sets become unbounded. The simplest example can be constructed as the union of a strip and a circle, whose diameter exceeds the width of the strip. Respectively, $\mathbf{f}_{\alpha}(\mu)$ is not infinite $\left[\mu_{0}, \boldsymbol{\rho}(G)\right]$. It follows from inequality

$$
\mathbf{I}_{\alpha}(G) \geqslant \frac{\pi \boldsymbol{\rho}(G)^{4}}{6}
$$

that the class of the domains obeying the condition $\boldsymbol{\rho}(G)<+\infty$ is the widest class, on which function (2.2) and functional (1.1) describe some geometric properties of the domain.

Since the sets $G(\mu)$ are monotonically embedded, this implies that $\mathbf{f}_{\alpha}(\mu)$ is a non-increasing function as $\alpha \geqslant 0$. In work [9], the identity was proved:

$$
\mathbf{f}_{2}(\mu)=\mathbf{i}_{2}(\mu)-2 \mu \mathbf{i}_{1}(\mu)
$$

where

$$
\mathbf{i}_{q}(\mu):=q \int_{\mu}^{\rho(G)} t^{q-1} \mathbf{a}(t) \mathrm{d} t, \quad q=1,2 .
$$

This representation implies the identities

$$
\left(\mathbf{f}_{2}(\mu)\right)^{\prime}=-2 \mathbf{i}_{1}(\mu), \quad\left(\mathbf{f}_{2}(\mu)\right)^{\prime \prime}=2 \mathbf{a}(\mu) .
$$

Thus, $\mathbf{f}_{2}(\mu)$ is twice differentiable, monotonically decreasing and strictly convex function. We also observe that almost everywhere there exists the third derivative of the function $\mathbf{f}_{2}(\mu)$, see [8].

Let us show that the function $\mathbf{f}_{\alpha}(\mu)$ possesses similar properties.

Lemma 2.1. Let $G$ be a simply-connected domain with a bounded Euclidean momentum of order $\alpha_{0}(>-1)$. Then $\mathbf{f}_{\alpha}(\mu)$ is a monotonically increasing function as $\alpha \geqslant 0$ and is strictly convex as $\alpha \geqslant 1$. This function is differentiable as $\alpha \geqslant 1$, is absolutely continuous as $\alpha \in(0,1)$, and if $\mathbf{l}(s)$ is a function of a bounded variation, then $\mathbf{f}_{\alpha}(\mu)$ is almost everywhere differentiable also as $0>\alpha>-1$.

Proof. Following [9], we observe that in the domain $G(\mu)$, the distance to its boundary is defined and we shall distinguish the levels sets of the domains $G$ and $G(\mu)$. It is easy to see that the distance to the boundary of the domain $G(\mu)$ is the function $\rho(x, G)-\mu$, therefore, $\mathbf{a}_{\mu}(s)=\mathbf{a}(s+\mu)(0 \leqslant s \leqslant \boldsymbol{\rho}(G(\mu)))$, where $\mathbf{a}_{\mu}(s)$ is the area of the level set of the function $\rho(x, G(\mu))$. Employing the definition of the Lebesgue integral and integrating by parts, it is easy to obtain the following representation:

$$
\begin{aligned}
\mathbf{f}_{\alpha}(\mu) & =\int_{0}^{\mathbf{a}(\mu)} s\left(\mathbf{a}_{\mu}\right)^{\alpha} \mathrm{d} \mathbf{a}_{\mu}=\alpha \int_{0}^{\rho(G(\mu))} s^{\alpha-1} \mathbf{a}_{\mu}(s) \mathrm{d} s \\
& =\alpha \int_{0}^{\rho(G)-\mu}(s+\mu)^{\alpha-1} \mathbf{a}(s+\mu) \mathrm{d} s=\alpha \int_{\mu}^{\rho(G)}(s-\mu)^{\alpha-1} \mathbf{a}(s) \mathrm{d} s,
\end{aligned}
$$


where $\alpha>0$. This representation implies the differentiability of the function as $\alpha \geqslant 1$ and the existence of the second derivative as $\alpha \geqslant 2$, as well as the identity:

$$
\begin{aligned}
& \left(\mathbf{f}_{\alpha}(\mu)\right)^{\prime}=-\alpha(\alpha-1) \int_{\mu}^{\rho(G)}(s-\mu)^{\alpha-2} \mathbf{a}(s) \mathrm{d} s=-\alpha \mathbf{f}_{\alpha-1}(\mu), \\
& \left(\mathbf{f}_{\alpha}(\mu)\right)^{\prime \prime}=\alpha(\alpha-1) \mathbf{f}_{\alpha-2}(\mu) .
\end{aligned}
$$

Particular cases of the latter identities are (2.4). Thus, $\mathbf{f}_{\alpha}(\mu)$ decreases monotonically as $\alpha \geqslant 1$ and is strictly convex as $\alpha \geqslant 2$.

Let us show that the above formulae hold under more general assumptions. We denote by $\mathbf{l}_{\mu}(s)$ the length of the level set of the function $\rho(x, G(\mu))$, then the identity $\mathbf{a}_{\mu}^{\prime}(s)=-\mathbf{l}_{\mu}(s)$ holds almost everywhere. Applying the formula of co-area [13] for the distance to the boundary, we obtain

$$
\mathbf{f}_{\alpha}(\mu)=\int_{0}^{\rho(G(\mu))} s^{\alpha} \mathbf{l}_{\mu}(s) \mathrm{d} s=\int_{\mu}^{\rho(G)}(s-\mu)^{\alpha} \mathbf{l}(s) \mathrm{d} s,
$$

where $\alpha>-1$. The latter representation implies the monotonicity of $\mathbf{f}_{\alpha}(\mu)$ as $\alpha \geqslant 0$ and another formula for the derivative:

$$
\mathbf{f}_{\alpha}^{\prime}(\mu)=-\alpha \int_{\mu}^{\rho(G)}(s-\mu)^{\alpha-1} \mathbf{l}(s) \mathrm{d} s .
$$

By this representation and the expression for the derivative we infer that identities (2.5) hold for $\alpha>0$ and $\alpha>1$, respectively.

Employing the non-negativity and measurability of the integrand and the Fubini theorem, for $\alpha>0$ and $0 \leqslant \nu \leqslant \rho(G)$ we obtain:

$$
\begin{aligned}
\int_{0}^{\nu} \mathbf{f}_{\alpha}^{\prime}(\mu) \mathrm{d} \mu & =-\alpha \int_{0}^{\nu} \mathrm{d} \mu \int_{\mu}^{\rho(G)}(s-\mu)^{\alpha-1} \mathbf{l}(s) \mathrm{d} s \\
& =-\alpha \int_{0}^{\nu} \mathbf{l}(s) \mathrm{d} s \int_{0}^{s}(s-\mu)^{\alpha-1} \mathrm{~d} \mu-\alpha \int_{\nu}^{\rho(G)} \mathbf{l}(s) \mathrm{d} s \int_{0}^{\nu}(s-\mu)^{\alpha-1} \mathrm{~d} \mu \\
& =-\int_{0}^{\nu} s^{\alpha} \mathbf{l}(s) \mathrm{d} s+\int_{\nu}^{\rho(G)}\left[(s-\nu)^{\alpha}-s^{\alpha}\right] \mathbf{l}(s) \mathrm{d} s \\
& =\int_{\nu}^{\rho(G)}(s-\nu)^{\alpha} \mathbf{l}(s) \mathrm{d} s-\int_{0}^{\rho(G)} s^{\alpha} \mathbf{l}(s) \mathrm{d} s=\mathbf{f}_{\alpha}(\nu)-\mathbf{f}_{\alpha}(0) .
\end{aligned}
$$

Hence, the function $\mathbf{f}_{\alpha}(\mu)$ is absolutely continuous as $\alpha>0$.

Assume that $\mathbf{l}(\mu)$ has a bounded variation. Then for $\alpha>-1$ we integrate by parts in (2.6) to obtain:

$$
\mathbf{f}_{\alpha}(\mu)=\frac{1}{\alpha+1} \int_{0}^{\rho(G)-\mu} \mathbf{l}(s+\mu) \mathrm{d} s^{\alpha+1}=\frac{1}{\alpha+1} \int_{\mu}^{\rho(G)} \mathbf{l}(s) \mathrm{d}(s-\mu)^{\alpha+1}
$$




$$
=\frac{\mathbf{l}(\boldsymbol{\rho}(G))(\boldsymbol{\rho}(G)-\mu)^{\alpha+1}}{\alpha+1}-\frac{1}{\alpha+1} \int_{\mu}^{\boldsymbol{\rho}(G)}(s-\mu)^{\alpha+1} \mathrm{~d} \mathbf{l}(s),
$$

where the latter integral is treated in the Riemann-Stiltjes sense and

$$
\mathbf{l}(\boldsymbol{\rho}(G)):=\lim _{\mu \rightarrow \boldsymbol{\rho}(G)} \mathbf{l}(\mu) .
$$

The assumptions of the lemma and the obtained representation yields that as $0 \geqslant \alpha>-1$, the derivative

$$
\mathbf{f}_{\alpha}^{\prime}(\mu)=-\mathbf{l}(\boldsymbol{\rho}(G))(\boldsymbol{\rho}(G)-\mu)^{\alpha}+\int_{\mu}^{\boldsymbol{\rho}(G)}(s-\mu)^{\alpha} \mathrm{d} \mathbf{l}(s)
$$

exists almost everywhere. In particular,

$$
(\mathbf{a}(\mu))^{\prime}=-\mathbf{l}(\boldsymbol{\rho}(G))+\int_{\mu}^{\rho(G)} \mathrm{d} \mathbf{l}(s)=-\mathbf{l}(\mu)
$$

almost everywhere.

In conclusion of the proof we provide simple examples of the domains, for which the function $\mathbf{l}(\mu)$ is not continuous. The domain with finitely many jumps of the function $\mathbf{l}(\mu)$ can be easily obtained by the well-known dumbbell domain, see [7]. Indeed, we consider the domain being the union of two same circles and a rectangle, whose width is less than the diameter of the circles. It is easy to confirm that in this case the considered function has exactly one jump. The domain with finitely many jumps can be obtained by union of the dumbbells so that they intersect by the handles of different width. A simpler example is the union of two rectangle of different widths forming a stair with two steps. In this case we also obtain exactly one jump for the function $\mathbf{l}(\mu)$. Increasing the number of the steps, we obtain finitely many or infinitely many jumps for the function.

Particular cases of monotonicity and convexity are two-sided estimates for the function $\mathbf{f}_{2}(\mu)$ and its derivative. However, these estimates are a special case of two-sided estimates obtained by means of Payne type inequality [9]

$$
\mathbf{I}_{2}(G) \leqslant \frac{2 \boldsymbol{\rho}(G)}{3}\left(\mathbf{I}_{1}(G)-\frac{\pi \boldsymbol{\rho}(G)^{3}}{12}\right) .
$$

Indeed, taking into consideration the first identity in $(2.4)$, we apply the latter inequality on the level sets $G(\mu)$ and we obtain the differential inequality:

$$
\mathbf{f}_{2}(\mu) \leqslant-\frac{2(\boldsymbol{\rho}(G)-\mu)}{3}\left(\frac{\left(\mathbf{f}_{2}(\mu)\right)^{\prime}}{2}+\frac{\pi(\boldsymbol{\rho}(G)-\mu)^{3}}{12}\right) .
$$

By simple algebraic arguments we can show that the latter inequality is equivalent to

$$
\left(\frac{\mathbf{f}_{2}(\mu)}{(\boldsymbol{\rho}(G)-\mu)^{3}}\right)^{\prime} \leqslant-\frac{\pi}{6}
$$

We integrate the obtained inequality over $[0, \mu]$ and $[\mu, \boldsymbol{\rho}(G)]$ and we get the two-sided estimate:

$$
\frac{2}{3} \boldsymbol{l}(\boldsymbol{\rho}(G))(\boldsymbol{\rho}(G)-\mu)^{3}+\frac{\pi}{6}(\boldsymbol{\rho}(G)-\mu)^{4} \leqslant \mathbf{f}_{2}(\mu) \leqslant\left(\frac{\mathbf{f}_{2}(0)}{\boldsymbol{\rho}(G)^{3}}-\frac{\pi \mu}{6}\right)(\boldsymbol{\rho}(G)-\mu)^{3},
$$

where $\boldsymbol{l}(\boldsymbol{\rho}(G))$ is the length of the level set of the function $\rho(x, G)$ separated by the distance $\mu=$ $\boldsymbol{\rho}(G)$ from the boundary of the domain. Applying then inequalities (2.4), by latter inequality we obtain easily two-sided estimates for $\mathbf{f}_{2}^{\prime}(\mu)$ and $\mathbf{f}_{2}^{\prime \prime}(\mu)$. In particular, these estimate imply a polynomial behavior of $\mathbf{f}_{2}(\mu)$ and its derivatives. 
To generalize the latter inequality, we rewrite the right inequality as

$$
\frac{1}{\boldsymbol{\rho}(G(\mu))^{3}}\left(\mathbf{f}_{2}(\mu)-\frac{\pi \boldsymbol{\rho}(G(\mu))^{4}}{6}\right) \leqslant \frac{1}{\boldsymbol{\rho}(G)^{3}}\left(\mathbf{f}_{2}(0)-\frac{\pi \boldsymbol{\rho}(G)^{4}}{6}\right) .
$$

We observe that

$$
\mathbf{I}_{2}(D)=\frac{\pi r^{4}}{6}
$$

where $D$ is a circle of radius $r$. This is why it is natural to consider the functional

$$
\mathbf{F}_{\alpha}(\mu):=\frac{1}{\boldsymbol{\rho}(G(\mu))^{\alpha+1}}\left(\mathbf{f}_{\alpha}(\mu)-\frac{2 \pi \boldsymbol{\rho}(G(\mu))^{\alpha+2}}{(\alpha+1)(\alpha+2)}\right) .
$$

In terms of the introduced notations, inequality $(2.9)$ casts into a simple form:

$$
\mathbf{F}_{2}(\mu) \leqslant \mathbf{F}_{2}(0) \text {. }
$$

Thus, it is natural to conjecture that similar inequality holds also for $\mathbf{F}_{\alpha}(\mu)$. Arguing in the same way, we obtain lower bounds:

$$
\mathbf{F}_{2}(\boldsymbol{\rho}(G)) \leqslant \mathbf{F}_{2}(\mu)
$$

and a corresponding conjecture for $\mathbf{F}_{\alpha}(\mu)$.

We are going to show that two latter inequalities are particular cases of the monotonicity of the function $\mathbf{F}_{\alpha}(\mu)$.

Theorem 2.1. Let $G$ be a simply-connected domain possessing a bounded Euclidean momentum of order $\alpha(>0)$. Then $\mathbf{F}_{\alpha}(\mu)$ is a monotonically decreasing function as $\alpha \geqslant 0$.

Corollary 1. Under assumption of Theorem 2.1, the function $\mathbf{f}_{\alpha}(\mu) \boldsymbol{\rho}(G(\mu))^{-(\alpha+1)}$ decreases monotonically on $[0, \boldsymbol{\rho}(G)]$. In particular, the inequality holds:

$$
\mathbf{f}_{\alpha}(\mu)<\left(1-\frac{\mu}{\boldsymbol{\rho}(G)}\right)^{\alpha+1} \mathbf{I}_{\alpha}(G),
$$

where $\mu \in(0, \boldsymbol{\rho}(G))$.

Another important corollary of Theorem 2.1 is a property on a power behavior of the functional $\mathbf{f}_{\alpha}(\mu)$.

Corollary 2. Let $G$ be a simply-connected domain and $\alpha \geqslant 0$. Then the inequalities hold:

$$
\begin{aligned}
& \mathbf{f}_{\alpha}(\mu) \leqslant\left(\mathbf{I}_{\alpha}(G)-\frac{2 \pi \mu \boldsymbol{\rho}(G)^{\alpha+1}}{(\alpha+1)(\alpha+2)}\right)\left(1-\frac{\mu}{\boldsymbol{\rho}(G)}\right)^{\alpha+1} \\
& \mathbf{f}_{\alpha}(\mu) \geqslant \frac{\boldsymbol{\rho}(G)^{\alpha+1}}{\alpha+1}\left(\boldsymbol{l}(\boldsymbol{\rho}(G))+\frac{2 \pi(\boldsymbol{\rho}(G)-\mu)}{\alpha+2}\right)\left(1-\frac{\mu}{\boldsymbol{\rho}(G)}\right)^{\alpha+1}
\end{aligned}
$$

where $0 \leqslant \mu \leqslant \boldsymbol{\rho}(G)$. Both inequalities become identity if and only if $G$ is a Bonnesen type domain.

As $\alpha=0$ and $\alpha=1$, the latter inequalities were proved in [9] and were applied for estimating the torsional rigidity and Euclidean momenta of domains with respect to the boundary.

It is obvious that inequality (2.11) generalizes Corollary 1. On the other hand, (2.12) implies the isoperimetric inequality

$$
\mathbf{f}_{\alpha}(\mu) \geqslant \frac{2 \pi \boldsymbol{\rho}(G)^{\alpha+2}}{(\alpha+1)(\alpha+2)}\left(1-\frac{\mu}{\boldsymbol{\rho}(G)}\right)^{\alpha+2},
$$

being opposite to the inequality in Corollary 1, and inequalty $(2.13)$ becomes identity if and only if $G$ is a circle. 
Corollary 2 can be reformulated as the following double inequality

$$
\begin{aligned}
& \frac{\boldsymbol{l}(\boldsymbol{\rho}(G)) \boldsymbol{\rho}(G)^{\alpha+1}}{\alpha+1}(\boldsymbol{\rho}(G)-\mu)^{\alpha+1} \leqslant \mathbf{f}_{\alpha}(\mu)-\frac{2 \pi(\boldsymbol{\rho}(G)-\mu)^{\alpha+2}}{(\alpha+1)(\alpha+2)} \\
& \leqslant\left(\mathbf{I}_{\alpha}(G) \boldsymbol{\rho}(G)^{-(\alpha+1)}-\frac{2 \pi \boldsymbol{\rho}(G)}{(\alpha+1)(\alpha+2)}\right)(\boldsymbol{\rho}(G)-\mu)^{\alpha+1}
\end{aligned}
$$

showing a power behavior explicitly, where the domain and the parameter $\alpha$ are fixed. Estimates for the derivatives of the function $\mathbf{f}_{\alpha}(\mu)$ are obtained by means of identities $(2.5)$.

Corollary 3. Let $G$ be a simply-connected domain and $\alpha \geqslant 1$. Then the inequalities hold:

$$
\begin{aligned}
& \mathbf{f}_{\alpha}^{\prime}(\mu) \geqslant-\alpha\left(\mathbf{I}_{\alpha-1}(G)-\frac{2 \pi \mu \boldsymbol{\rho}(G)^{\alpha}}{\alpha(\alpha+1)}\right)\left(1-\frac{\mu}{\boldsymbol{\rho}(G)}\right)^{\alpha} \\
& \mathbf{f}_{\alpha}^{\prime}(\mu) \leqslant-\boldsymbol{\rho}(G)^{\alpha}\left(\boldsymbol{l}(\boldsymbol{\rho}(G))+\frac{2 \pi(\boldsymbol{\rho}(G)-\mu)}{\alpha+1}\right)\left(1-\frac{\mu}{\boldsymbol{\rho}(G)}\right)^{\alpha},
\end{aligned}
$$

where $0 \leqslant \mu \leqslant \boldsymbol{\rho}(G)$. Both inequalities become identities if and only if $G$ is a Bonnesen type domain.

Corollary 4. Let $G$ be a simply-connected domain and $\alpha \geqslant 2$. Then the inequalities hold:

$$
\begin{aligned}
& \mathbf{f}_{\alpha}^{\prime \prime}(\mu) \leqslant \alpha(\alpha-1)\left(\mathbf{I}_{\alpha-2}(G)-\frac{2 \pi \mu \boldsymbol{\rho}(G)^{\alpha-1}}{\alpha(\alpha-1)}\right)\left(1-\frac{\mu}{\boldsymbol{\rho}(G)}\right)^{\alpha-1}, \\
& \mathbf{f}_{\alpha}^{\prime \prime}(\mu) \geqslant(\alpha-1) \boldsymbol{\rho}(G)^{\alpha-1}\left(\boldsymbol{l}(\boldsymbol{\rho}(G))+\frac{2 \pi(\boldsymbol{\rho}(G)-\mu)}{\alpha}\right)\left(1-\frac{\mu}{\boldsymbol{\rho}(G)}\right)^{\alpha-1},
\end{aligned}
$$

where $0 \leqslant \mu \leqslant \boldsymbol{\rho}(G)$. Both inequalities become identities if and only if $G$ is a Bonnesen type domain.

It follows from [10] and Theorem 2.1 that the functional

$$
\mathbf{E}(\alpha, \mu):=(\alpha+1) \mathbf{F}_{\alpha}(\mu)
$$

is a monotonically decreasing function in both its arguments. At that, the monotonicity in the first argument is called an isoperimetric monotonicity in the parameter $\alpha$. Indeed, fixing $\mu$, we obtain a relation between various geometric characteristics of the set $G(\mu)$ in a form of an inequality. On the other hand, fixing $\alpha$, we obtain inequalities similar, for instance, to the monotonicity of the functional $\mathbf{a}(\mu)$. In fact, Corollary 3 provides a quantitative variation of the derivation of the functional.

If instead of the function $\rho(x, G)$ we consider a classical warping function of a domain and pass from the class of simply-connected domains to the class of finitely-connected domains on the plane, it turns out that we can prove statements similar to the above ones. In this case, the base results are those obtained in works [1, 11, 14]. In what follows we formulate the results omitting their detailed discussion.

Let $G$ be a finitely-connected domain on the plane. We denote by $\Gamma_{0}$ the external boundary component of the boundary $\partial G$, while $\Gamma_{1}, \ldots, \Gamma_{n}$ stand for the internal components of the boundary. A warping function of the domain $G$ is the unique solution $u(x, G)$ of the following boundary value problem

$$
\left\{\begin{array}{l}
\Delta u=-2 \text { in } G, \\
u=0 \text { on } \Gamma_{0}, \\
u=c_{i} \text { on } \Gamma_{i}, \quad i=1, \ldots, n,
\end{array}\right.
$$

where the constants $c_{i}$ are determined by the condition

$$
\oint_{\Gamma_{i}} \frac{\partial u}{\partial n} \mathrm{~d} s=-2 a_{i}, \quad i=1, \ldots, n .
$$


Here $\partial / \partial n$ denotes the derivative along the internal normal and $a_{i}$ be the area enveloped by the curve $\Gamma_{i}$.

We denote by $G_{0}$ the domain containing $G$ with a boundary coinciding with $\Gamma_{0}$. We extend continuously the function $u(x, G)$ by the constants in the sets enveloped by the curves $\Gamma_{i}$, $i=1, \ldots, n$. At that, we keep the same notation for the extension. We consider the following integral functional of the domain

$$
\mathbf{T}_{\beta}(G):=\int_{G_{0}} u(x, G)^{\beta} \mathrm{dA},
$$

where $\beta>-1$. In the case of a simply-connected domain and as $\beta=1$, the latter functional coincides with the torsional rigidity of the domain $G$ up to a multiplicative constant.

We denote by $G(\nu)$ the level set of the function $u(x, G)$, that is,

$$
G(\nu):=\left\{x \in G_{0} \mid u(x, G)>\nu\right\} .
$$

We note that as a part of the boundary of the set $G(\nu)$, the curves $\Gamma_{i}$ can serve. For the domain with the functional $\mathbf{T}_{\beta}(G)(\beta<+\infty)$, all level set have finite area as $\nu<\mathbf{u}(G)$, see [14], where $\mathbf{u}(G):=\sup _{x \in G} u(x, G)$.

Similar to Euclidean momenta, we consider the functional

$$
\phi_{\beta}(\nu):=\mathbf{T}_{\beta}(G(\nu)),
$$

where $0 \leqslant \nu \leqslant \mathbf{u}(G), \beta>-1$.

Lemma 2.2. Let $G$ be a finitely-connected domain with a bounded functional $\mathbf{T}_{\beta}(G)(\beta \geqslant 0)$. Then $\phi_{\beta}(\mu)$ is a monotonically decreasing function as $\beta \geqslant 0$ and is strictly convex as $\beta \geqslant 1$, and also is absolutely continuous as $\beta>0$.

Proof. By the definition of the function $u(x, G)$ and the sets $G(\nu)$ we establish easily the identity

$$
u(x, G(\nu))=u(x, G)-\nu(x \in G(\nu)),
$$

in particular, $\mathbf{u}(G(\nu))=\mathbf{u}(G)-\nu$. Applying the definition of the Lebesgue integral, we obtain

$$
\boldsymbol{\phi}_{\beta}(\nu)=\int_{0}^{\mathbf{A}(G(\nu))} t\left(\mathbf{a}_{\nu}\right)^{\beta} \mathrm{d} \mathbf{a}_{\nu}=\int_{\nu}^{\mathbf{u}(G)}(t-\nu)^{\beta} \mathrm{d} \mathbf{a}(t)+\sum_{c_{i}>\nu}\left(c_{i}-\nu\right)^{\beta} a_{i} .
$$

We apply the formula of co-area [13] to the function $u(x, G)$ to get:

$$
\boldsymbol{\phi}_{\beta}(\nu)=\int_{\nu}^{\mathbf{u}(G)}(t-\nu)^{\beta} \boldsymbol{\ell}(t) \mathrm{d} t+\sum_{c_{i}>\nu}\left(c_{i}-\nu\right)^{\beta} a_{i}
$$

where

$$
\boldsymbol{\ell}(t):=\int_{\Gamma(t)} \frac{\mathrm{d} s}{|\nabla u(x, G)|}
$$

and $\Gamma(t)=\{x \in G \mid u(x, G)=t\}$. The obtained formula implies a monotonous decreasing of $\phi_{\beta}(\nu)$ as $\beta \geqslant 0$.

Formula 2.16) implies also the identity

$$
\phi_{\beta}^{\prime}(\nu)=-\beta \int_{\nu}^{\mathbf{u}(G)}(t-\nu)^{\beta-1} \ell(t) \mathrm{d} t-\beta \sum_{c_{i}>\nu}\left(c_{i}-\nu\right)^{\beta-1} a_{i}=-\beta \boldsymbol{\phi}_{\beta-1}(\nu) .
$$

This yields a strict convexity of $\boldsymbol{\phi}_{\beta}(\nu)$ as $\beta \geqslant 1$. The absolute continuity of the function $\boldsymbol{\phi}_{\beta}(\nu)$ can be proved in the same way as in Lemma 2.1.

Following work [14], similar to definition 2.10, we consider the functional

$$
\boldsymbol{\Phi}_{\beta}(\nu):=\frac{1}{\mathbf{u}(G(\nu))^{\beta}}\left(\phi_{\beta}(\nu)-\frac{2 \pi \mathbf{u}(G(\nu))^{\beta+1}}{\beta+1}\right),
$$


where $\beta \geqslant 0$. In the case, where the domain $G$ is contained in a concentric annulus, it is well-known that the expression in the brackets vanish identically, while in other cases, Payne showed [6] that this expression is strictly positive.

The following analogue of Theorem 2.1 holds true.

Theorem 2.2. Let $G$ be a finitely-connected domain with a bounded functional $\mathbf{T}_{\beta}(G)$ for some $\beta(\geqslant 0)$. Then $\boldsymbol{\Phi}_{\beta}(\nu)$ is monotonically decreasing as $\beta \geqslant 1$.

We also provide some corollaries indicating a power behavior of the function $\phi_{\beta}(\nu)$.

Corollary 5. Under the assumptions of Theorem 2.2, the inequalities

$$
0 \leqslant \boldsymbol{\phi}_{\beta}(\nu)-\frac{2 \pi \mathbf{u}(G)^{\beta+1}}{\beta+1}\left(1-\frac{\nu}{\mathbf{u}(G)}\right)^{\beta+1} \leqslant \mathbf{T}_{\beta}(G)\left(1-\frac{\nu}{\mathbf{u}(G)}\right)^{\beta},
$$

hold, where $0 \leqslant \nu \leqslant \mathbf{u}(G)$. Both inequalities become identities if and only if $G$ is a concentric annulus.

Corollary 6. Let $G$ be a finitely-connected domain and $\beta \geqslant 1$. Then the inequalities hold

$$
\phi_{\beta}^{\prime}(\nu) \geqslant-\left(\beta \mathbf{T}_{\beta-1}(G)-2 \pi \nu \mathbf{u}(G)^{\beta-1}\right)\left(1-\frac{\nu}{\mathbf{u}(G)}\right)^{\beta-1}, \quad \phi_{\beta}^{\prime}(\nu) \leqslant-2 \pi(\mathbf{u}(G)-\nu)^{\beta},
$$

where $0 \leqslant \nu \leqslant \mathbf{u}(G)$. Both inequalities become identities if and only if $G$ is a concentric annulus.

Corollary 7. Let $G$ be a finitely-connected domain and $\beta \geqslant 2$. Then the inequalities hold $\boldsymbol{\phi}_{\beta}^{\prime \prime}(\nu) \leqslant \beta\left((\beta-1) \mathbf{T}_{\beta-2}(G)-2 \pi \nu \mathbf{u}(G)^{\beta-2}\right)\left(1-\frac{\nu}{\mathbf{u}(G)}\right)^{\beta-2}, \quad \boldsymbol{\phi}_{\beta}^{\prime \prime}(\nu) \geqslant 2 \pi \beta(\mathbf{u}(G)-\nu)^{\beta-1}$, where $0 \leqslant \nu \leqslant \mathbf{u}(G)$. Both inequalities become identities if and only if $G$ is a concentric annulus.

\section{PROOF OF MAIN Results}

Proof of Theorem 2.1. The statement of Lemma 2.1 on absolute continuity of the function $\mathbf{f}_{\alpha}(\mu)$ implies that the decreasing of $\mathbf{F}_{\alpha}(\mu)$ is equivalent to the inequality $\mathbf{F}_{\alpha}^{\prime}(\mu) \leqslant 0$ for almost each $\mu \in(0, \boldsymbol{\rho}(G))$. In view of the identity $\boldsymbol{\rho}(G(\mu))=\boldsymbol{\rho}(G)-\mu$ and Definition (2.10), the latter inequality is equivalent to the estimate

$$
\left(\frac{\mathbf{f}_{\alpha}(\mu)}{(\boldsymbol{\rho}(G)-\mu)^{\alpha+1}}\right)^{\prime} \leqslant-\frac{2 \pi}{(\alpha+1)(\alpha+2)} .
$$

As $\alpha=2$, this inequality coincides with (2.8), therefore, the statement of the theorem in this particular case has been justified earlier.

In works 9, 10, there were studied the properties of the functional $(\alpha+1) \mathbf{F}_{\alpha}(0)$ as of the function of the argument $\alpha$. A Bonnesen type domain is a convex domain being the union of two semi-circles and a rectange, in particular, as the rectangle degenerates, we obtain a circle. Since this statement is a key one in the proof, we formulate it in terms of our notations.

Theorem A. [10] Let $G$ be a simply-connected domain and $\mathbf{I}_{p_{0}}(G)<+\infty$ for some $p_{0} \in$ $[-1, \infty)$. Then

1) if $G$ does not coincide with an extremal in the Bonnesen inequality, then $(\alpha+1) \mathbf{F}_{\alpha}(0)$ is a strictly decreasing function of $\alpha$,

2) if $G$ coincides with one of the extremals in the Bonnesen inequality, then $(\alpha+1) \mathbf{F}_{\alpha}(0) \equiv$ $\mathbf{l}(\boldsymbol{\rho}(G))$ as $\alpha \in[-1,+\infty)$. 
In particular, the inequality

$$
(\alpha+1) \mathbf{F}_{\alpha}(0) \leqslant \alpha \mathbf{F}_{\alpha-1}(0)
$$

holds true. The inequality becomes an identity if and only if $G$ is a Bonnesen type domain. An important fact is that all level set of the distance function of a Bonnesen type domain also envelop a Bonnesen type domain.

We apply the latter inequality on the level sets $G(\mu)$. In view of definition $(2.2)$, the inequality casts into the form

$$
\mathbf{f}_{\alpha}(\mu) \leqslant \frac{\alpha \boldsymbol{\rho}(G(\mu))}{\alpha+1} \mathbf{f}_{\alpha-1}(\mu)-\frac{2 \pi \boldsymbol{\rho}(G(\mu))^{\alpha+2}}{(\alpha+1)^{2}(\alpha+2)} .
$$

Taking into consideration identities (2.5), after simple algebraic transformations we obtain that the latter inequality is equivalent to inequality (3.1). This completes the proof.

Proof of Corollary 2. Inequality (2.11) is equivalent to inequality

$$
\mathbf{F}_{\alpha}(\mu) \leqslant \mathbf{F}_{\alpha}(0)
$$

being a direct corollary of Theorem 2.1.

We again employ the fact that the functional $(\alpha+1) \mathbf{F}_{\alpha}(0)$ is a monotonically increasing function of $\alpha$. On the other hand, it was proved in work [9] that

$$
\lim _{\alpha \rightarrow+\infty} \frac{\alpha \mathbf{I}_{\alpha}(G)}{\boldsymbol{\rho}(G)^{\alpha+1}}=\boldsymbol{l}(\boldsymbol{\rho}(G)) .
$$

These two statements yield the following isoperimetric inequality

$$
\mathbf{I}_{\alpha}(G) \geqslant \frac{\boldsymbol{\rho}(G)^{\alpha+1}}{\alpha+1}\left(\boldsymbol{l}(\boldsymbol{\rho}(G))+\frac{2 \pi \boldsymbol{\rho}(G)}{p+2}\right) .
$$

We apply the obtained inequality on the level sets $G(\mu)$ as well as the identity $\boldsymbol{\rho}(G(\mu))=$ $\boldsymbol{\rho}(G)-\mu$ and we arrive at inequality $(2.12$.

Proof of Theorem 2.2. The statement of Lemma 2.2 on absolute continuity of the function $\phi_{\beta}(\nu)$ yields that the decreasing of $\boldsymbol{\Phi}_{\beta}(\nu)$ is equivalent to $\boldsymbol{\Phi}_{\beta}^{\prime}(\nu) \leqslant 0$ for almost each $\nu \in$ $(0, \mathbf{u}(G))$. In view of the identity $\mathbf{u}(G(\nu))=\mathbf{u}(G)-\nu$ and corresponding definitions, the latter inequality is equivalent to the estimate

$$
\left(\frac{\phi_{\beta}(\nu)}{(\mathbf{u}(G)-\nu)^{\beta}}\right)^{\prime} \leqslant-\frac{2 \pi}{\beta+1} .
$$

In our case, a key ingredient in the proof is a statement from work [14. We provide this statement in a form adapted to our notations.

Theorem B. Let $G$ be a finitely-connected domain, $\mathbf{T}_{p_{0}}(G)<+\infty$ for some $p_{0} \in[0, \infty)$. Then

1) If $G$ is not a concentric annulus, then $\boldsymbol{\Phi}_{\beta}(0)(\mathbf{u}(G))^{-1}$ is a strictly increasing function in $\beta$ as $\beta \geqslant p_{0}$.

2) If $G$ is a concentric annulus, then $\boldsymbol{\Phi}_{\beta}(0)(\mathbf{u}(G))^{-1} \equiv 0$ for $\beta \in[0,+\infty)$.

In particular, the inequality holds true:

$$
\boldsymbol{\Phi}_{\beta}(0) \leqslant \boldsymbol{\Phi}_{\beta-1}(0) .
$$

The inequality becomes an identity only for a concentric annulus. We observe that the level sets of the warping function of a concentric annulus also envelop a concentric annulus.

Applying the latter inequality on the level sets $G(\nu)$, we obtain

$$
\phi_{\beta}(\nu) \leqslant \mathbf{u}(G(\nu)) \phi_{\beta-1}(\nu)-\frac{2 \pi \mathbf{u}(G(\nu))^{\beta+1}}{\beta(\beta+1)} .
$$

Employing (2.17), it is easy to establish that inequality (3.4) is equivalent to inequality (3.2). The proof is complete. 
Proof of Corollary 5. The left inequality in the statement is the Payne inequality for $G(\nu)$, while the right inequality is equivalent to inequality $\boldsymbol{\Phi}_{\beta}(\nu) \leqslant \boldsymbol{\Phi}_{\beta}(0)$.

In conclusion we note that in this case, an analogue of functional $(2.14)$ is the functional

$$
\mathbf{R}(\beta, \nu):=\boldsymbol{\Phi}_{\beta}(\nu)
$$

monotone in both variables. A monotonous behavior in the free parameter $\beta$ was proved in work [14]. As for the distance to the boundary, this monotonicity is isoperimetric.

The author thanks an anonymous referee for valuable remarks and comments on the work.

\section{BIBLIOGRAPHY}

1. R.G. Salakhudinov. Integral properties of the classical warping function of a simply connected domain // Matem. Zamet. 92:3, 447-458 (2012). [Math. Notes. 92:3, 412-421 (2012).]

2. F.G. Avkhadiev. Solution of the generalized Saint Venant problem // Matem. Sborn. 189:12, 3-12 (1998). [Sb. Math. 189:12, 1739-1748 (1998).]

3. F.G. Avkhadiev. Hardy type inequalities in higher dimensions with explicit estimate of constants // Lobachevskii J. Math. 21, 3-31 (2006).

4. R. Bañuelos, M. van den Berg, and T. Carroll. Torsional rigidity and expected lifetime of brownian motion // J. London Math. Soc. 66:2, 499-512 (2002).

5. R.G. Salakhudinov. Two-sided estimates for the Lp-norms of the stress function for convex domains in $\mathbb{R}^{n}$ // Izv. VUZov. Matem. 3, 41-49 (2006). [Russ. Math. Iz. VUZ. 50:3, 39-46 (2006).]

6. L.E. Payne. Some inequalities in the torsion problem for multiply connected regions // in "Studies in Mathematical analysis and Related Topics" . Stanford Univ. Press, Stanford, California, 270280 (1962).

7. G. Pólya, G. Szegö. Isoperimetric inequalities in mathematical physics. Ann. Math. Stud. 27. Princeton Univ. Press, Princeton (1951).

8. C. Bandle. Isoperimetric inequalities and applications. Pitman Advanced Publishing Program, Boston (1980).

9. R.G. Salakhudinov. Refined inequalities for euclidian moments of a domain with respect to its boundary // SIAM J. Math. Anal. 44:4, 2949-2961 (2012).

10. R.G. Salakhudinov. Isoperimetric properties of Euclidean boundary moments of a simply connected domain // Izv. VUZov. Matem. 8, 66-79 (2013). [Russ. Math. Iz. VUZ. 57,:8, 57-69 (2013).]

11. R.G. Salakhudinov. Isoperimetric inequalities for $l^{p}$-norms of the stress function of a multiply connected plane domain // Izv. VUZov. Matem. 9, 75-80 (2013). [Russ. Math. Iz. VUZ. 57:9, 62-66 (2013).]

12. R.G. Salahudinov. Isoperimetric inequalities for $l^{p}$-norms of the distance function to the boundary // Uchen. Zap. Kazan. Univ. Ser. Fiz.-Mat. Nauki. 148:2, 151-162 (2006). (in Russian).

13. J. Maly, D. Swanson, and W. Ziemer. The coarea formula for sobolev mappings // Trans. Amer. Math. Soc. 355:2, 477-492, 012002.

14. R.G. Salakhudinov. Payne type inequalities for $l^{p}{ }^{-n o r m s}$ of the warping functions // J. Math. Anal. Appl. 410:2, 659-669 (2014).

Rustem Gumerovich Salakhudinov,

Kazan Federal University,

Lobachevsky Institute of Mathematics and Mechanics

Kazan Federal University,

Kremlevskaya str., 18,

420008, Kazan, Russia

E-mail: rsalakhud@gmail.com 